\title{
ON THE UPPER AND LOWER SEMICONTINUITY OF THE AUMANN INTEGRAL
}

\author{
Nicholas C. YANNELIS* \\ University of Illinois at Urbana-Champaign, Champaign, IL 61820, USA
}

Submitted September 1988, accepted July 1989

\begin{abstract}
Let $(T, \tau, \mu)$ be a finite measure space, $X$ be a Banach space, $P$ be a metric space and let $L_{1}(\mu, X)$ denote the space of equivalence classes of $X$-valued Bochner integrable functions on $(T, \tau, \mu)$. We show that if $\phi: T \times P \rightarrow 2^{X}$ is a set-valued function such that for each fixed $p \in P, \phi(\cdot, p)$ has a measurable graph and for each fixed $t \in T, \phi(t, \cdot)$ is either upper or lower semicontinuous then the Aumann integral of $\phi$, i.e., $\int_{T} \phi(t, p) \mathrm{d} \mu(t)=\left\{\int_{T} x(t) \mathrm{d} \mu(t): x \in S_{\phi}(p)\right\}$, where $S_{\phi}(p)=$ $\left\{y \in L_{1}(\mu, X): y(t) \in \phi(t, p) \mu\right.$-a.e. $\}$, is either upper or lower semicontinuous in the variable $p$ as well. Our results generalize those of Aumann $(1965,1976)$ who has considered the above problem for $X=R^{n}$, and they have useful applications in general equilibrium and game theory.
\end{abstract}

\section{Introduction}

Let $(T, \tau, \mu)$ be a finite measure space, $P$ be a metric space, $X$ be a Banach space and let $L_{1}(\mu, X)$ denote the space of equivalence classes of $X$-valued Bochner integrable functions on $(T, \tau, \mu)$. Let $\phi: T \times P \rightarrow 2^{X}$ be a set-valued function (where $2^{X}$ denotes the set of all non-empty subsets of $X$ ), such that for each fixed $p \in P, \phi(\cdot, p)$ has a measurable graph and for each fixed $t \in T, \phi\left(t,,^{-}\right)$is either upper or lower semicontinuous. We wish to know whether the Aumann integral of $\phi$, i.e., $\int_{T} \phi(t, p) \mathrm{d} \mu(t)=\left\{\int_{T} x(t) \mathrm{d} \mu(t): x \in S_{\phi}(p)\right\}$, where $S_{\phi}(p)=\left\{y \in L_{1}(\mu, X): y(t) \in \phi(t, p) \mu\right.$-a.e. $\}$, is either upper or lower semicontinuous in the variable $p$ as well. It is the purpose of this paper to provide an answer to the above question. Specifically, we show (Theorems 3.1 and 3.2) that integration preserves upper semicontinuity (u.s.c.) and that (Theorem 3.3) integration preserves lower semicontinuity (1.s.c.).

We will first discuss briefly the main applications of our results in general equilibrium and game theory. The question of whether integration preserves

*This is a revised version of my paper entitled 'On the Lebesgue-Aumann Dominated Convergence Theorem in Infinite Dimensional Spaces', written in 1986. The present version has benefitted from the comments, discussions and suggestions of Erik Balder, M. Ali Khan, JeanFrancois Mertens, Aldo Rustichini and of a knowledgeable referee. My debt to the pioneering works of Aumann (1965) and Debreu (1967) should be evident. Of course, I am responsible for any remaining shortcomings. 
u.s.c. arises naturally in general equilibrium. In particular, $T$ denotes the measure space of agents, $X$ denotes the commodity space, $P$ denotes the price space, $\phi(t, p)$ denotes the demand set of agent $t$ at prices $p$ and the integral of $\phi$ denotes the aggregate demand set. For $X=R^{\ell}$ (where $R^{\ell}$ is the $\ell$-fold Cartesian product of the set of real numbers $R$ ), Aumann (1965, 1966, 1976) has examined the above problem which is fundamental in order to prove the existence of an equilibrium for an economy with a measure space of agents and with finitely many commodities. However, if one wishes to examine the problem of the existence of an equilibrium for an economy with a measure space of agents and with an infinite dimensional commodity space, then an infinite dimensional generalization of the above result seems to be required. ${ }^{1}$ It turns out that the finite dimensional arguments of Aumann cannot be readily adopted to cover Banach-valued correspondences. ${ }^{2}$ In particular, his proof is based on the Lyapunov theorem, a result which is false in infinite dimensional spaces. Nevertheless, for strong forms of u.s.c. correspondences (i.e., weakly u.s.c. correspondences) results analogous to those of Aumann have been obtained in Yannelis (1988).

It is important to note that the method of proof in Yannelis (1988) utilizes in a crucial way the convex valuedness of the set-valued function. In particular, the argument is based on the 'approximate version of the Lyapunov-Richter Theorem', and it is applied to convex, closed valued and weakly u.s.c. correspondences. ${ }^{3}$ Since the correspondences we consider in Theorem 3.1 are not convex valued or closed valued or weakly u.s.c. this argument cannot be applied here. Nevertheless, in the setting of Yannelis we prove a result (Theorem 3.2) similar to his, using a weak sequential convergence theorem obtained in Yannelis (1989).

Theorem 3.2 has found useful applications in game theory [see for instance Khan (1986) or Yannelis (1987) among others]. Specifically, the main result needed to prove the existence of a Nash equilibrium for a game with a continuum of players, is that the set of integrable selections of the set-valued

\footnotetext{
${ }^{1}$ It should be noted that Debreu (1967, pp. 364-367) has already examined the problem of whether integration preserves u.s.c. or l.s.c. for correspondences taking values in a Banach space. Moreover, Debreu provided an extension of the Lebesgue dominated convergence to infinite dimensional spaces. However, his notion of convergence is different than the one used in this paper [as in Aumann (1965) we follow the Kuratowski-type of convergence of sets, see the next section of the paper for a definition], and consequently our results do not follow from his.

${ }^{2} \mathrm{~A}$ correspondence is a set-valued function for which all image sets are non-empty.

${ }^{3}$ To be more specific, by the 'approximate version of the Lyapunov-Richter Theorem' we mean that if $F$ is a set-valued function from the atomless measure space $T$ to the separable Banach space $X$, then under appropriate assumptions $\mathrm{cl} f F=\int \overline{\operatorname{con}} F$ (where cl denotes norm closure and $\overline{c o n}$ denotes closed convex hull). This result is proved by Datko (1973) for $X$ being a reflexive separable Banach space. The reflexivity assumption was relaxed by Khan (1985). However, recently Rustichini and Yannelis (1988) have shown that the norm closure can be removed if the dimensionality of the measure space is larger than the dimensionality of the separable Banach space. In particular, this occurs if the economy has 'many more' agents than commodities. Of course the concept of dimension has to be given a rigorous formulation.
} 
function $\phi: T \times P \rightarrow 2^{X}$, i.e., $S_{\phi}(p)$ is weakly u.s.c. whenever for each $t \in T, \phi(t, p)$ is weakly u.s.c. with respect to $p$. Now, if $\pi: L_{1}(\mu, X) \rightarrow X$ is a linear mapping defined by $\pi(x)=\int_{t \in T} x(t) \mathrm{d} \mu(t)$, the integral of the set-valued function $\phi: T \times P \rightarrow 2^{X}$ is $\pi\left(S_{\phi}(p)\right)=\left\{\pi(x): x \in S_{\phi}(p)\right\}$. Hence, if $S_{\phi}(\cdot)$ is weakly u.s.c. so is $\pi\left(S_{\phi}(\cdot)\right)=\int \phi(t, \cdot)$. It is exactly this idea which is exploited in the proof of Theorem 3.2 in order to show that integration preserves weak u.s.c.

Finally, we wish to note that the problem of whether integration preserves l.s.c. (Theorem 3.3) has been useful in the literature of games with incomplete information in order to prove the I.s.c. of expectation operators [see for instance Balder-Yannelis (1988)].

The paper proceeds as follows: Section 2 contains notation and definitions. Our main results are stated in section 3 and their proofs are collected in section 4 .

\section{Notation and definitions}

\subsection{Notation}

$R^{n}$ denotes the $n$-fold Cartesian product of the set of real numbers $R$.

$\overline{\operatorname{con}} A$ denotes the closed convex hull of set $A$.

$2^{A}$ denotes the set of all non-empty subsets of the set $A$.

$\varnothing$ denotes the empty set.

$\checkmark$ denotes the set theoretic subtraction.

dist denotes distance.

proj denotes projection.

If $A \subset X$, where $X$ is a Banach space, $\mathrm{cl} A$ denotes the norm closure of $A$.

If $F_{n}(n=1,2, \ldots)$ is a sequence of non-empty subsets of a Banach space $X$, we will denote by $L s F_{n}$ and $L i F_{n}$ the set of its (strong) limit superior and (strong) limit inferior points respectively, i.e.,

$$
\begin{aligned}
& L s F_{n}=\left\{x \in X: x=\lim _{k \rightarrow \infty} x_{n_{k}}, x_{n_{k}} \in F_{n_{k}}, k=1,2, \ldots\right\}, \quad \text { and } \\
& L i F_{n}=\left\{x \in X: x=\lim _{n \rightarrow \infty} x_{n}, x_{n} \in F_{n}, n=1,2, \ldots\right\} .
\end{aligned}
$$

A $w$ in front of $L s F_{n}\left(L i F_{n}\right)$ will mean limit superior (limit inferior) with respect to the weak topology $\sigma\left(X^{*}, X\right)$.

\subsection{Definitions}

Let $X$ and $Y$ be sets. The graph of the set-valued function $\phi: X \rightarrow 2^{Y}$ is 
denoted by $G_{\phi}=\{(x, y) \in X \times Y: y \in \phi(x)\}$. Let $(T, \tau, \mu)$ be a complete, finite measure space, and $X$ be a separable Banach space. The set-valued function $\phi: T \rightarrow 2^{X}$ is said to have a measurable graph if $G_{\phi} \in \tau \otimes \beta(X)$, where $\beta(X)$ denotes the (norm) Borel $\sigma$-algebra on $X$ and $\otimes$ denotes product $\sigma$-algebra. The correspondence $\phi: T \rightarrow 2^{X}$ is said to be lower measurable if for every open subset $V$ of $X$, the set $\{t \in T: \phi(t) \cap V \neq \varnothing\}$ is an element of $\tau$. Recall [see for instance Debreu $(1967$, p. 359$)$ or Himmelberg $\left(1975\right.$, p. 47)] that if $\phi: T \rightarrow 2^{X}$ has a measurable graph, then $\phi$ is lower measurable. Furthermore, if $\phi(\cdot)$ is closed valued and lower measurable then $\phi: T \rightarrow 2^{X}$ has a measurable graph. A well-known result of Aumann (1967) which will be of fundamental importance in this paper, [see also Himmelberg (1975, Theorem 5.2, p. 60)] says that if $(T, \tau, \mu)$ is a complete, finite measure space, $X$ is a separable metric space and $\phi: T \rightarrow 2^{X}$ is a non-empty valued correspondence having a measurable graph, then $\phi(\cdot)$ admits a measurable selection, i.e., there exists a measurable function $f: T \rightarrow X$ such that $f(t) \in \phi(t) \mu$-a.e.

We now define the notion of a Bochner integrable function. We will follow closely Diestel and Uhl (1977). Let $(T, \tau, \mu)$ be a finite measure space and $X$ be a Banach space. A function $f: T \rightarrow X$ is called simple is there exist $x_{1}, x_{2}, \ldots, x_{n}$ in $X$ and $\alpha_{1}, \alpha_{2}, \ldots, \alpha_{n}$ in $\tau$ such that $f=\sum_{i=1}^{n} x_{i} \chi_{\alpha_{i}}$, where $\chi_{\alpha_{i}}(t)=1$ if $t \in \alpha_{i}$ and $\chi_{\alpha_{i}}(t)=0$ if $t \notin \alpha_{i}$. A function $f: T \rightarrow X$ is said to be $\mu$-measurable if there exists a sequence of simple functions $f_{n}: T \rightarrow X$ such that $\lim _{n \rightarrow \infty}\left\|f_{n}(t)-f(t)\right\|=0$ for almost all $t \in T$. A $\mu$-measurable function $f: T \rightarrow X$ is said to be Bochner integrable if there exists a sequence of simple functions $\left\{f_{n}: n=1,2, \ldots\right\}$ such that

$$
\lim _{n \rightarrow \infty} \int_{T}\left\|f_{n}(t)-f(t)\right\| \mathrm{d} \mu(t)=0
$$

In this case we define for each $E \in \tau$ the integral to be $\int_{E} f(t) \mathrm{d} \mu(t)=$ $\lim _{n \rightarrow \infty} \int_{E} f_{n}(t) \mathrm{d} \mu(t)$. It can be shown [see Diestel and Uhl (1977, Theorem 2, p. 45)] that, if $f: T \rightarrow X$ is a $\mu$-measurable function then $f$ is Bochner integrable if and only if $\int_{T}\|f(t)\| \mathrm{d} \mu(t)<\infty$. We denote by $L_{1}(\mu, X)$ the space of equivalence classes of $X$-valued Bochner integrable functions $x: T \rightarrow X$ normed by

$$
\|x\|=\int_{T}\|x(t)\| \mathrm{d} \mu(t)
$$

It is a standard result that normed by the functional $\|\cdot\|$ above, $L_{1}(\mu, X)$ becomes a Banach space [see Diestel and Uhl (1977, p. 50)].

We denote by $S_{\phi}$ the set of all $X$-valued Bochner integrable selections from $\phi: T \rightarrow 2^{X}$, i.e., 


$$
S_{\phi}=\left\{x \in L_{1}(\mu, X): x(t) \in \phi(t) \mu-\text { a.e. }\right\} \text {. }
$$

Moreover, as in Aumann (1965) the integral of the set-valued function $\phi: T \rightarrow 2^{X}$ is defined as follows:

$$
\int_{T} \phi(t) \mathrm{d} \mu(t)=\left\{\int_{T} x(t) \mathrm{d} \mu(t): x \in S_{\phi}\right\}
$$

In the sequel we will denote the above integral by $\int \phi$. Recall that the set-valued function $\phi: T \rightarrow 2^{X}$ is said to be integrably bounded if there exists a map $h \in L_{1}(\mu, R)$ such that $\sup \{\|x\|: x \in \phi(t)\} \leqq h(t) \mu$-a.e. Moreover, note that if $T$ is a complete measure space, $X$ is a separable Banach space and $\phi: T \rightarrow 2^{X}$ is an integrably bounded, non-empty valued correspondence having a measurable graph, then by the Aumann measurable selection theorem we can conclude that $S_{\phi}$ is non-empty and therefore $\int_{T} \phi(t) \mathrm{d} \mu(t)$ is non-empty as well.

Let $A_{n}(n=1,2, \ldots)$ be a sequence of non-empty subsets of a Banach space. Following Kuratowski (1966, p. 339) we say that $A_{n}$ converges in $A$ (written as $A_{n} \rightarrow A$ ) if and only if $L i A_{n}=L s A_{n}=A$. It may be useful to remind the reader that $L i A_{n}$ and $L s A_{n}$ are both closed sets and that $L i A_{n} \subset L s A_{n}$ [see Kuratowski (1966, pp. 336-338)].

Let $X$ be a metric space and $Y$ be a Banach space. The set-valued function $\phi: X \rightarrow 2^{Y}$ is said to be u.s.c. at $x_{0} \in X$, if for any neighborhood $N\left(\phi\left(x_{0}\right)\right)$ of $\phi\left(x_{0}\right)$, there exists a neighborhood $N\left(x_{0}\right)$ of $x_{0}$ such that for all $x \in N\left(x_{0}\right), \phi(x) \subset N\left(\phi\left(x_{0}\right)\right)$. We say that $\phi$ is u.s.c. if $\phi$ is u.s.c. at every point $x \in X$. Recall that this definition is equivalent to the fact that the set $\{x \in X: \phi(x) \subset V\}$ is open in $X$ for every open subset $V$ of $Y$ [see for instance Kuratowski (1966, Theorem 3, p. 176)].

Let $v$ be a small positive number and let $B$ be the open unit ball in $Y$. The set-valued function $\phi: X \rightarrow 2^{Y}$ is said to be quasi upper-semicontinuous (q.u.s.c.) at $x \in X$, if whenever the sequence $x_{n}(n=1,2, \ldots)$ in $X$ converges to $x$, then for some $n_{0}, \phi\left(x_{n}\right) \subset \phi(x)+v B$ for all $n \geqq n_{0}$. We say that $\phi$ is q.u.s.c. if $\phi$ is q.u.s.c. at every point $x \in X$. It can be easily checked [see for instance Aubin and Ekeland $(1984$, p. 108)] that if $\phi$ is compact valued, quasi uppersemicontinuity implies upper-semicontinuity and vice-versa.

In particular, note that if $K$ is a compact subset of $Y$, the subsets $N(K, \delta)=\{y \in Y: \operatorname{dist}(K, y) \leqq \delta\}$ form a fundamental basis of neighborhoods of $K$, i.e., any neighborhood of the compact set $K$ contains $N(K, \delta)$ for a suitable $\delta>0$. Consequently, if the correspondence $\phi: X \rightarrow 2^{Y}$ is compact valued, then $\phi$ is u.s.c. at $x_{0} \in X$ if and only if

for every $\delta>0$, there exists $v>0$ such that for all 


$$
x \in N\left(x_{0}, v\right), \phi(x) \subset N\left(\phi\left(x_{0}\right), \delta\right) .
$$

However, if for $x_{0} \in X, \phi\left(x_{0}\right)$ is not compact valued, then for all $x \in N\left(x_{0}, v\right)$, $\phi(x) \subset N\left(\phi\left(x_{0}\right), \delta\right)$ may hold, even if $\phi$ is not u.s.c. To see this define the set-valued function $\phi: R \rightarrow 2^{R^{2}}$ by $\phi(z)=\{(x, y): x=z\}$. Obviously (2.1) holds for $\delta=v$, i.e., $\phi$ is $q$. u.s.c. but $\phi$ is not u.s.c. Indeed, the set $\{(x, y):|y|<1 /|x|\}$ is a neighborhood of $\phi(0)$, but for any $x \neq 0, \phi(x)$ is not contained in the set $\{(x, y):|y|<1 /|x|\}$.

Let now $P$ and $X$ be metric spaces. The set-valued function $F: P \rightarrow 2^{x}$ is said to be 1.s.c. if the sequence $p_{n}(n=1,2, \ldots)$ in $P$ converges to $p \in P$, then $F(p) \subset \operatorname{Li} F\left(p_{n}\right)$. Finally recall that the correspondence $F: P \rightarrow 2^{X}$ is said to be continuous if and only if it is u.s.c. and 1.s.c.

With all these preliminaries out of the way we can now turn to the statements of the main theorems.

\section{The main theorems}

Below we now state our main results:

Theorem 3.1. Let $(T, \tau, \mu)$ be a complete, finite measure space, $P$ be a metric space and $X$ be a separable Banach space. Let $\psi: T \times P \rightarrow 2^{X}$ be a non-empty valued, integrably bounded correspondence, such that for each fixed $t \in T$, $\psi\left(t,{ }^{\cdot}\right)$ is q.u.s.c. and for each fixed $p \in P, \psi(\cdot, p)$ has a measurable graph. Then

$$
\int \psi(t, \cdot) \text { is q.u.s.c. }
$$

Theorem 3.2. Let $(T, \tau, \mu)$ be a complete, finite measure space, $P$ be a metric space and $X$ be a separable Banach space. Let $\psi: T \times P \rightarrow 2^{X}$ be a non-empty, closed, convex valued correspondence such that:

(i) for each fixed $t \in T, \psi(t, \cdot)$ has a weakly closed graph, ${ }^{4}$

(ii) for all $(t, p) \in T \times P, \quad \psi(t, p) \subset K(t)$ where $K: T \rightarrow 2^{X}$ is an integrably bounded, weakly compact and non-empty valued correspondence.

Then

$\int \psi(t, \cdot)$ has a weakly closed graph.

Theorem 3.3. Let $(T, \tau, \mu)$ be a complete, finite measure space, $X$ be a separable Banach space and $P$ be a metric space. Let $\phi: T \times P \rightarrow 2^{X}$ be an integrably bounded set-valued function such that for each fixed $t \in T, \phi\left(t,,^{\cdot}\right)$ is l.s.c. and for each fixed $p \in P, \phi(\cdot, p)$ has a measurable graph. Then

${ }^{4}$ By this we mean that if the sequence $\left\{p_{n}: n=1,2, \ldots\right\}$ in $P$ converges to $p \in P$ then $w-L s \psi\left(t, p_{n}\right) \subset \psi(t, p)$ for each $t \in T$. 
$\int \phi(t, \cdot)$ is l.s.c.

Remark 3.1. If in addition to the assumptions of Theorem 3.1, it is assumed that $\int \psi\left(t,,^{\cdot}\right)$ is compact valued, then we can conclude that $\int \psi(t, \cdot)$ is u.s.c.

Remark 3.2. If in Theorem 3.1 we add the assumption that $\psi(\cdot, \cdot)$ is convex valued and that for all $(t, p) \in T \times P, \psi(t, p) \subset K$, where $K$ is a weakly compact, convex, non-empty subset of $X$, then it follows from Lemma 4.1 (see next section) that $\int_{T} \psi(t, \cdot) \mathrm{d} \mu(t)$ is weakly compact valued and we can conclude that $\int_{T} \psi(t, \cdot) \mathrm{d} \mu(t)$ is weakly u.s.c., i.e., the set $\left\{p \in P: \int_{T} \psi(t, p) \mathrm{d} \mu(t) \subset V\right\}$ is open in $P$ for every weakly open subset $V$ of $X$. Hence, from Theorem 3.1 we can obtain a version of Theorem 4.1 in Yannelis (1988) which does not require $(T, \tau, \mu)$ to be atomless. Note, however, that the measurability assumption made in Theorem 3.1 is slightly stronger than the one made in Theorem 4.1 of Yannelis (1988) (recall Lemma 4.4 in the next section).

Remark 3.3. It can be easily shown by means of the failure of the Lyapunov theorem in infinite dimensional spaces that Theorem 3.2 is false without the convex valuedness of the set-valued function $\psi: T \times P \rightarrow 2^{X}$ [see Rustichini (1989) for a complete argument]. Hence, the convexity assumption on the values of $\psi$ cannot be dropped from the similar result to Theorem 3.2 obtained by Yannelis (1988).

The Corollaries below follow directly from Theorems 3.1, 3.3 and Remark 3.1. They extend some results of Aumann (1965, Theorem 5 and Corollary 5.2) to separable Banach spaces.

Corollary 3.1. Let $(T, \tau, \mu)$ be a complete, finite measure space, $P$ be a metric space and $X$ be a separable Banach space. Let $\psi: T \times P \rightarrow 2^{X}$ be an integrably bounded, non-empty valued correspondence such that for each fixed $p \in P$, $\psi(\cdot, p)$ has a measurable graph and for each fixed $t \in T, \psi(t, \cdot)$ is continuous. Moreover, suppose that $\int_{T} \psi(t, \cdot) \mathrm{d} \mu(t)$ is compact valued. Then

$$
\int_{T} \psi(t, \cdot) \mathrm{d} \mu(t) \text { is continuous. }
$$

Corollary 3.2. (Lebesgue-Aumann Dominated Convergence Theorem). ${ }^{5}$ Let $(T, \tau, \mu)$ be a complete, finite measure space and $X$ be a separable Banach space. Let $\phi_{n}: T \rightarrow 2^{X} \quad(n=1,2, \ldots)$ be a sequence of integrably bounded, nonempty valued correspondence having a measurable graph, such that:

${ }^{5}$ Compare with Theorem 6.3, in Debreu (1967, p. 366) and with Corollary 3.2 in Yannelis (1989) where different notions of convergence of sequences of set-valued functions were used.. 
(i) For all $n(n=1,2, \ldots), \phi_{n}(t) \subset K \mu$-a.e., where $K$ is a compact, non-empty subset of $X$, and

(ii) $\phi_{n}(t) \rightarrow \phi(t) \mu$-a.e.

Then

$$
\int_{T} \phi_{n}(t) \mathrm{d} \mu(t) \rightarrow \operatorname{cl} \int_{T} \phi(t) \mathrm{d} \mu(t)
$$

Moreover, if $\phi(\cdot)$ is convex valued then

$$
\int_{T} \phi_{n}(t) \mathrm{d} \mu(t) \rightarrow \int_{T} \phi(t) \mathrm{d} \mu(t)
$$

\section{Proof of the main theorems}

\subsection{Lemmata}

For the proof of our main results we will need some preparatory lemmata.

Lemma 4.1. Let $(T, \tau, \mu)$ be a finite measure space and $X$ be a Banach space. Let $\phi: T \rightarrow 2^{X}$ be a set-valued function satisfying the following condition:

(i) $\phi(t) \subset K \mu$-a.e., where $K$ is a weakly compact, non-empty subset of $X$. Then

$$
\operatorname{cl} \int_{T} \overline{\operatorname{con}} \phi(t) \mathrm{d} \mu(t)=\int_{T} \overline{\operatorname{con}} \phi(t) \mathrm{d} \mu(t) .
$$

Proof. Let $\tilde{K}=\overline{\operatorname{con}} K$. Note that $\tilde{K}$ is weakly compact, [see Dunford and Schwartz (1958, Theorem 4, p. 434)] non-empty and convex. Hence, from Diestel's theorem [Diestel (1977, Theorem 2)] we have that $S_{\tilde{K}}$ is weakly compact in $L_{1}(\mu, X)$. Since $\overline{\operatorname{con}} \phi(\cdot)$ is norm closed and convex valued so is $S_{\overline{c o n} \phi}$. It is a consequence of the Separation Theorem that the weak and norm topologies coincide on closed convex sets. Hence, $S_{\overline{\operatorname{con}} \phi}$ is weakly closed. Since $S_{\overline{\operatorname{con}} \phi} \subset S_{\tilde{K}}$ and the latter set is weakly compact we can conclude that $S_{\overline{c o n} \phi}$ is weakly compact. Define the mapping $\gamma: L_{1}(\mu, X) \rightarrow X$ by $\gamma(x)=$ $\int_{T} x(t) \mathrm{d} \mu(t)$. Certainly $\gamma$ is linear and norm continuous. It follows from Iheorem 15 in Dunford and Schwartz (1958, p. 422) that $\gamma$ is also weakly continuous. Therefore, $\gamma\left(S_{\overline{\operatorname{con}} \phi}\right)=\left\{\gamma(x): x \in S_{\operatorname{con} \phi}\right\}=\int_{\mathrm{T}} \overline{\operatorname{con}} \phi(t) \mathrm{d} \mu(t)$ is weakly compact, and we can conclude that $\operatorname{cl} \int_{T} \overline{\operatorname{con}} \phi(t) \mathrm{d} \mu(t)=\int_{T} \overline{\operatorname{con}} \phi(t)$. This completes the proof of Lemma 4.1.

Notice that the above proof of Lemma 4.1 showed that $\int_{T} \overline{\operatorname{con}} \phi(t) \mathrm{d} \mu(t)$ is weakly compact. Hence, Lemma 4.1 may be seen as the infinite dimensional 
extension of Theorem 4 of Aumann (1965). Also, note that Datko (1973) and Khan (1985) showed that if $(T, \tau, \mu)$ is assumed to be atomless then the conclusion of Lemma 4.1 can be strengthened to $\mathrm{cl} \int \phi=\int \overline{\operatorname{con}} \phi$.

The result below is an infinite dimensional $L i$ version of the Fatou Lemma for the set of all integrable selections from a correspondence.

Lemma 4.2. Let $(T, \tau, \mu)$ be a complete, finite measure space and $X$ be a separable Banach space. Let $\phi_{n}: T \rightarrow 2^{X}(n=1,2 \ldots)$ be a sequence of integrably bounded set-valued functions having a measurable graph, i.e., $G_{\phi_{n}} \in \tau \otimes \beta(X)$, Then

$$
S_{L i \phi_{n}} \subset L i S_{\phi_{n}}
$$

Proof. See Yannelis (1989, Lemma 5.3).

Lemma 4.3. Let $(T, \tau, \mu)$ be a finite measure space and $X$ be a separable Banach space. Let $\left\{f_{n}: n=1,2, \ldots\right\}$ be a sequence of functions in $L_{p}(\mu, X), 1 \leqq$ $p<\infty$ such that $f_{n}$ converges weakly to $f \in L_{p}(\mu, X)$. Suppose that for all $n$ $(n=1,2, \ldots), f_{n}(t) \in F(t) \mu$-a.e., where $F: T \rightarrow 2^{X}$ is a weakly compact, integrably bounded, non-empty valued correspondence. Then

$$
f(t) \in \overline{\operatorname{con}} w-L s\left\{f_{n}(t)\right\} \mu-\text { a.e. }
$$

Proof. See Yannelis (1989, Corollary 3.1).

Lemma 4.4. Let $(T, \tau, \mu)$ be a complete finite measure space and $X$ be a separable Banach space. Let $\left\{F_{n}: n=1,2, \ldots\right\}$ be a sequence of non-empty valued and lower measurable correspondences. Then $\operatorname{LiF}_{n}(\cdot)$ has a measurable graph, i.e., $G_{L i F_{n}} \in \tau \otimes \beta(X)$.

Proof. First notice that $\operatorname{LiF}_{n}(\cdot)$ is closed valued [recall from Kuratowski (1966, pp. 336-337), that if $A_{n}$ is a sequence of sets, $L i A_{n}$ and $L s A_{n}$ are both closed sets]. By definition [see Kuratowski (1966, p. 335)], LiF $(t)=$ $\left\{f \in X: \lim _{n \rightarrow \infty} \operatorname{dist}\left(f, F_{n}(t)\right)=0\right\}$. Since by assumption the sequence of setvalued functions $F_{n}(\cdot)$ have a measurable graph and $(T, \tau, \mu)$ is a complete measure space, $F_{n}(\cdot)$ are lower measurable. It follows from Himmelberg (1975, Theorem 3.3, p. 50) that $\operatorname{dist}\left(f, F_{n}(t)\right)$ is continuous in $f$ and measurable in $t$, i.e., dist $(\cdot, \cdot)$ is jointly measurable with respect to the $\sigma$ algebra $\tau \otimes \beta(X)$. Hence, $\lim _{n \rightarrow \infty} \operatorname{dist}\left(f, F_{n}(t)\right)$ is jointly measurable with respect to the $\sigma$-algebra $\tau \otimes \beta(X)$. Notice that 


$$
G_{L i F_{n}}=\left\{(t, f) \in T \times X: \lim _{n \rightarrow \infty} \operatorname{dist}\left(f, F_{n}(t)\right)=0\right\}
$$

Since $\lim _{n \rightarrow \infty} \operatorname{dist}\left(f, F_{n}(t)\right)$ is jointly measurable, the set $G_{L i F_{n}}$ belongs to $\tau \otimes \beta(X)$, i.e., $I_{i} i F_{n}$ has a measurable graph. This completes the proof of Lemma 4.4.

Remark 4.1. Under the assumptions of Lemma $4.4 L s F_{n}(\cdot)$ has a measurable graph as well. Simply notice that [see Kuratowski (1966, p. 337)] $L s F_{n}(t)=\left\{f \in X: L i \operatorname{dist}\left(f, F_{n}(t)\right)=0\right\}$.

\subsection{Proof of Theorem 3.1}

Without loss of generality we may assume throughout the argument that $\int_{T} \mathrm{~d} \mu(t)=1$. Let $B$ be the open unit ball in $X$, and $v$ be a small positive number. We must show that if $\left\{p_{n}: n=1,2, \ldots\right\}$ is a sequence in $P$ converging to $p \in P$, then for a suitable $n_{0}$,

$$
\int_{T} \psi\left(t, p_{n}\right) \mathrm{d} \mu(t) \subset \int_{T} \psi(t, p) \mathrm{d} \mu(t)+v B \quad \text { for } \quad \text { all } n \geqq n_{0} .
$$

Define the set-valued function $S_{\psi}: P \rightarrow 2^{L_{1}(\mu, X)}$ by $S_{\psi}(p)=$ $\left\{x \in L_{1}(\mu, X): x(t) \in \psi(t, p) \mu-\right.$ a.e. $\}$. Let $B$ and $\widetilde{B}$ be the open unit balls in $X$ and $L_{1}(\mu, X)$, respectively. We first show that for a suitable $n_{0}, S_{\psi}\left(p_{n}\right) \subset$ $S_{\psi}(p)+v \widetilde{B}$ for all $n \geqq n_{0}$.

We begin by finding the suitable $n_{0}$. Since for each fixed $t \in T, \psi(t, \cdot)$ is q.u.s.c. we can find a minimal $M_{t}$ such that

$$
\psi\left(t, p_{n}\right) \subset \psi(t, p)+\delta B \quad \text { for all } n \geqq M_{t}
$$

where $\delta=v / 3 \mu(T)$.

We now show that $M_{t}$ is a measurable function of $t$. However, first we make a few observations. By assumption for each fixed $p$ and $n$, $G_{\psi\left(\cdot, p_{n}\right)+\delta B} \in \tau \otimes \beta(X)$ and so does $\left(G_{\psi\left(\cdot, p_{n}\right)+\delta B}\right)^{c}$ (where $S^{c}$ denotes the complement of the set $S)$. It is easy to see that $\left.G_{\psi(\cdot, p)} \cap G_{\psi\left(\cdot, p_{n}\right)+\delta B}\right)^{\mathrm{c}} \in \tau \otimes$ $\beta(X)$. Therefore, the set

$$
U=\left\{(t, x) \in T \times X:(t, x) \in G_{\psi(\cdot, p)} \cap\left(G_{\psi\left(\cdot, p_{n}\right)+\delta B}\right)^{\mathbf{c}}\right\}
$$

belongs to $\tau \otimes \beta(X)$.

It follows from the projection theorem [see for instance Debreu (1967, (3.4), p. 357)] that

$$
\operatorname{proj}_{T}(U) \in \tau .
$$


Notice that

$$
\begin{aligned}
\operatorname{proj}_{T}(U) & =\left\{t \in T: \psi(t, p) \not \psi \psi\left(t, p_{n}\right)+\delta B\right\} \\
& =\left\{t \in T: \psi(t, p) \backslash\left(\psi\left(t, p_{n}\right)+\delta B\right) \neq \varnothing\right\} .
\end{aligned}
$$

By virtue of the measurability of the above set we can now conclude that $M_{t}$ is a measurable function of $t$. In particular, simply notice that

$$
\begin{aligned}
\left\{t \in T: M_{t}=m\right\}= & \bigcap_{n \geqq m}\left\{t \in T: \psi\left(t, p_{n}\right) \subset \psi(t, p)+\delta B\right\} \\
& \cap\left\{t \in T: \psi\left(t, p_{m-1}\right) \not \subset \psi(t, p)+\delta B\right\} .
\end{aligned}
$$

We are now in a position to choose the desired $n_{0}$. Since $\psi(\cdot, \cdot)$ is integrably bounded there exists $h \in L_{1}(\mu, R)$ such that for almost all $t \in T, \sup \{\|x\|$ $: x \in \psi(t, p)\} \leqq h(t)$ for each $p \in P$.

Choose $\delta_{1}$ such that if $\mu(S)<\delta_{1},(S \subset T)$, then $\int_{S} h(t) \mathrm{d} \mu(t)<v / 3$. Since $M_{t}$ is a measurable function of $t$, we can choose $n_{0}$ such that $\mu\left(\left\{t \in T: M_{t} \geqq n_{0}\right\}\right)<$ $\delta_{1}$. This is the desired $n_{0}$. Let $n \geqq n_{0}$ and $y \in S_{\psi}\left(p_{n}\right)$. We must show that $y \in S_{\psi}(p)+v \tilde{B}$.

By assumption, for each fixed $p \in P, \psi(\cdot, p)$ has a measurable graph and $\psi(\cdot, \cdot)$ is non-empty valued. Hence, by the Aumann measurable selection theorem there exists a measurable function $f_{1}: T \rightarrow X$ such that $f_{1}(t) \in \psi(t, p) \mu$-a.e. Define the correspondence $\theta: T \rightarrow 2^{X}$ by $\theta(t)=(\{y(t)\}+\delta B) \cap \psi(t, p)$. It follows from (4.1) that for all $t \in T_{0}=\left\{t: M_{t} \leqq\right.$ $\left.n_{0}\right\}, \theta(t) \neq \varnothing$. Moreover, $\theta(\cdot)$ has a measurable graph. Another application of the Aumann measurable selection theorem allows us to guarantee the existence of a measurable function $f_{2}: T \rightarrow X$ such that $f_{2}(t) \in \theta(t) \mu$-a.e. Define $f: T \rightarrow X$ by

$$
f(t)=\left\{\begin{array}{lll}
f_{1}(t) & \text { for } & t \notin T_{0} \\
f_{2}(t) & \text { for } & t \in T_{0}
\end{array}\right.
$$

Then $f(t) \in \psi(t, p) \mu$-a.e. and since $\psi(\cdot, \cdot)$ is integrably bounded we can conclude that $f \in S_{\psi}(p)$. If we show that $\|f-y\|<v$ then $y \in S_{\psi}(p)+v \tilde{B}$ and we will be done. But this is easy to see. We have

$$
\begin{aligned}
\|f-y\| & =\int_{T / T_{0}}\left\|f_{1}(t)-y(t)\right\| \mathrm{d} \mu(t)+\int_{T_{0}}\left\|f_{2}(t)-y(t)\right\| \mathrm{d} \mu(t) \\
& <2 \int_{T / T_{0}} h(t) \mathrm{d} \mu(t)+\int_{T_{0}} \delta \mathrm{d} \mu(t)
\end{aligned}
$$




$$
<2 v / 3+\delta \mu(T)=2 v / 3+v / 3 \mu(T) \cdot \mu(T)=v
$$

This completes the proof of the fact that, if the sequence $\left\{p_{n}: n=1,2, \ldots\right\}$ in $P$ converges to $p \in P$, then for a suitable $n_{0}$,

$$
S_{\psi}\left(p_{n}\right) \subset S_{\psi}(p)+v \widetilde{B} \quad \text { for } \text { all } n \geqq n_{0} .
$$

Define now the mapping $\gamma: L_{1}(\mu, X) \rightarrow X$ by $\gamma(x)=\int_{T} x(t) \mathrm{d} \mu(t)$. It follows from (4.2) that for all $n \geqq n_{0}$,

$$
\begin{aligned}
\gamma\left(S_{\psi}\left(p_{n}\right)\right) & =\left\{\gamma(x): x \in S_{\psi}\left(p_{n}\right)\right\} \\
& =\int_{T} \psi\left(t, p_{n}\right) \mathrm{d} \mu(t) \subset \gamma\left(S_{\psi}(p)+v \widetilde{B}\right)=\gamma\left(S_{\psi}(p)\right)+\gamma(v \widetilde{B}) \\
& =\int_{T} \psi(t, p) \mathrm{d} \mu(t)+v B .
\end{aligned}
$$

Hence,

$$
\int_{T} \psi\left(t, p_{n}\right) \mathrm{d}_{\mu}(t) \subset \int_{T} \psi(t, p) \mathrm{d} \mu(t)+v B \quad \text { for } \quad \text { all } n \geqq n_{0} .
$$

i.e., $\int_{T} \psi(t, \cdot) \mathrm{d} \mu(t)$ is q.u.s.c. as was to be shown.

\subsection{Proof of Theorem 3.2}

We first show that the set-valued function $S_{\psi}: P \rightarrow 2^{L_{1}(\mu, X)}$ defined by

$$
S_{\psi}(p)=\left\{x \in L_{1}(\mu, X): x(t) \in \psi(t, p) \mu-\text { a.e. }\right\}
$$

has a weakly closed graph, i.e., if $\left\{p_{n}: n=1,2, \ldots\right\}$ is a sequence in $P$ converging to $p \in P$, then

$$
w-L s S_{\psi}\left(p_{n}\right) \subset S_{\psi}(p)
$$

To this end let $x \in w-L s S_{\psi}\left(p_{n}\right)$, i.e., there exists $x_{k}(k=1,2, \ldots)$ in $L_{1}(\mu, X)$ such that $x_{k}$ converges weakly to $x \in L_{1}(\mu, X)$, and $x_{k}(t) \in \psi\left(t, p_{n_{k}}\right) \mu-$ a.e., we must show that $x \in S_{\psi}(p)$. It follows from Lemma 4.3 that $x(t) \in \overline{\operatorname{con}} w-$ $L s\left\{x_{k}(t)\right\} \mu-$ a.e. and therefore,

$$
x(t) \in \overline{\operatorname{con}} w-L s \psi\left(t, p_{n}\right) \mu-\text { a.e. }
$$


Since for each fixed $t \in T, \psi(t, \cdot)$ has a weakly closed graph we have that

$$
w-\operatorname{Ls} \psi\left(t, p_{n}\right) \subset \psi(t, p) \mu-\text { a.e. }
$$

Combining (4.4) and (4.5) and taking into account the fact that $\psi$ is convex valued we have that $x(t) \in \psi(t, p) \mu$-a.e. Since $\psi$ is integrably bounded, we can conclude that $x \in S_{\psi}(p)$. This completes the proof of the fact that $S_{\psi}(\cdot)$ has a weakly closed graph. Define the linear mapping

$$
\pi: L_{1}(\mu, X) \rightarrow X \text { by } \pi(x)=\int x(t) \mathrm{d} \mu(t)
$$

It follows from (4.3) that if the sequence $\left\{p_{n}: n=1,2, \ldots\right\}$ in $P$ converges to $p \in P$, then

$$
\begin{aligned}
\pi\left(w-L s S_{\psi}\left(p_{n}\right)\right) & =\left\{\pi(x): x \in w-L s S_{\psi}\left(p_{n}\right)\right\}=w-L s \int \psi\left(t, p_{n}\right) \subset \pi\left(S_{\psi}(p)\right) \\
& =\left\{\pi(x): x \in S_{\psi}(p)\right\} \\
& =\int \psi(t, p),
\end{aligned}
$$

i.e., $\int \psi(t, \cdot)$ has a weakly closed graph as was to be shown.

\subsection{Proof of Theorem 3.3}

We first show that the set-valued function $S_{\phi}: P \rightarrow 2^{L_{1}(\mu, X)}$ defined by

$$
S_{\phi}(p)=\left\{y \in L_{1}(\mu, X): y(t) \in \phi(t, p) \mu-\text { a.e. }\right\}
$$

is l.s.c.

To see this, let $\left\{p_{n}: n=1,2, \ldots\right\}$ be a sequence in $P$ converging to $p \in P$. We must show that $S_{\phi}(p) \subset L i S_{\phi}\left(p_{n}\right)$. Since by assumption for each fixed $t \subset T$, $\phi(t, \cdot)$ is l.s.c. we have that $\phi(t, p) \subset \operatorname{Li} \phi\left(t, p_{n}\right)$ for all $t \in T$, and therefore,

$$
S_{\phi}(p) \subset S_{L i \phi}\left(p_{n}\right)
$$

It follows now from Lemma 4.2 that (4.6) can be written as

$$
S_{\phi}(p) \subset S_{L i \phi}\left(p_{n}\right) \subset \operatorname{Li} S_{\phi}\left(p_{n}\right)
$$

Hence, 


$$
S_{\phi}(\cdot) \text { is 1.s.c. }
$$

Define now the mapping $\gamma: L_{1}(\mu, X) \rightarrow X$ by $\gamma(x)=\int_{T} x(t) \mathrm{d} \mu(t)$. Then $\gamma$ is linear and norm continuous. Notice that

$$
\gamma\left(S_{\phi}(p)\right)=\left\{\gamma(x): x \in S_{\phi}(p)\right\}=\int_{T} \phi(t, p) \mathrm{d} \mu(t) .
$$

Since $S_{\phi}(\cdot)$ is 1.s.c. so is $\gamma\left(S_{\phi}\right)$, i.e., $\int_{T} \phi(t, \cdot) \mathrm{d} \mu(t)$ is 1. s.c. as was to be shown. This completes the proof of Theorem 3.2.

\subsection{Proof of Corollary 3.2}

We begin by proving an approximate version of the Fatou Lemma in infinite dimensions [see also Balder (1988), Khan and Majumdar (1986) and Yannelis (1988) for $w-L s$ versions of this Lemma], which may be considered as an extension of the finite dimensional Fatou-type lemmata obtained in Aumann (1965), Artstein (1979), Balder (1984), Hildenbrand and Mertens (1971), Rustichini and Yannelis (1986), and Schmeidler (1970).

Lemma 4.5. Let $(T, \tau, \mu)$ be a complete, finite measure space and $X$ be $a$ separable Banach space. Let $\phi_{n}: T \rightarrow 2^{X}(n=1,2, \ldots)$ be a sequence of nonempty valued, graph measurable and integrably bounded correspondences, taking values in a compact, non-empty subset of $X$. Then

$$
L s \int_{T} \phi_{n}(t) \mathrm{d} \mu(t) \subset \operatorname{cl} \int_{T} L s \phi_{n}(t) \mathrm{d} \mu(t)
$$

Moreover, if $\operatorname{Ls} \phi_{n}(\cdot)$ is convex valued, then

$$
L s \int_{T} \phi_{n}(t) \mathrm{d} \mu(t) \subset \int_{T} L s \phi_{n}(t) \mathrm{d} \mu(t) .
$$

Proof. Denote by $P$ the interval $[0,1)$. As in Rustichini and Yannelis (1986) define the set-valued function $\psi: T \times P \rightarrow 2^{X}$ by

$$
\psi(t, p)=\left\{\begin{array}{lll}
\phi_{n}(t) & \text { if } \quad 1 /(n+1)<p<1 / n, \\
\phi_{n}(t) \cup \phi_{n+1}(t) & \text { if } \quad p=1 /(n+1), \\
L s \phi_{n}(t) & \text { if } \quad p=0 .
\end{array}\right.
$$

It can be easily checked that for each fixed $t \in T, \psi(t, \cdot)$ is u.s.c. and that for each fixed $p \in P, \psi(\cdot, p)$ has a measurable graph [recall Lemma 4.4 and 
Remark 4.1]. Moreover, $\psi$ is integrably bounded. Hence, $\psi$ satisfies all the assumptions of Theorem 3.1. and thus, $\int_{T} \psi(t, \cdot) \mathrm{d} \mu(t)$ is q.u.s.c. Let now $x \in L s \int_{T} \phi_{n}(t) \mathrm{d} \mu(t)$, i.e., there exists $x_{n_{k}}$ such that $\lim _{k \rightarrow \infty} x_{n_{k}}=x$, $x_{n_{k}} \in \int_{T} \phi_{n_{k}}(t) \mathrm{d} \mu(t)(k=1,2, \ldots)$. We wish to show that $x \in \operatorname{cl} \int_{\mathrm{T}} L s \phi_{n}(t) \mathrm{d} \mu(t)$.

Since $\int_{T} \psi(t, \cdot) \mathrm{d} \mu(t)$ is q.u.s.c. it follows that if $p_{n_{k}}$ converges to 0 then $\int_{T} \psi\left(t, p_{n_{k}}\right) \mathrm{d} \mu(t) \subset \int_{T} \psi(t, 0) \mathrm{d} \mu(t)+v B$ for all sufficiently large $k$. Consequently, $x_{n_{k}} \in \int_{T} \psi(t, 0) \mathrm{d} \mu(t)+v B$ for all sufficiently large $k$ and therefore,

$$
x \in \mathrm{cl} \int_{T} \psi(t, 0) \mathrm{d} \mu(t) \equiv \mathrm{cl} \int_{T} L s \phi_{n}(t) \mathrm{d} \mu(t)
$$

as was to be shown. If now $L s \phi_{n}(\cdot)$ is convex valued (recall that $L s \phi_{n}(\cdot)$ is closed valued as well) it follows from Lemma 4.1 and the first conclusion of Lemma 4.5 that

$$
L s \int_{T} \phi_{n} \mathrm{~d} \mu(t) \subset \operatorname{cl} \int_{T} L s \phi_{n}(t) \mathrm{d} \mu(t)=\int_{T} L s \phi_{n}(t) \mathrm{d} \mu(t) .
$$

The proof of Lemma 4.5 is now complete.

We are now ready to complete the proof of Corollary 3.2. Notice first that it follows from Lemma 4.2 that

$$
\int \operatorname{Li} \phi_{n} \subset \operatorname{Li} \int \phi_{n}
$$

To see this define the linear mapping $\gamma: L_{1}(\mu, X) \rightarrow X$ by $\gamma(x)=\int_{T} x(t) \mathrm{d} \mu(t)$. Note that $\gamma\left(S_{L i \phi_{n}}\right)=\left\{\gamma(x): x \in S_{L i \phi_{n}}\right\}=\int L i \phi_{n}$ and hence by virtue of Lemma 4.2 we can conclude that

$$
\gamma\left(S_{L i \phi_{n}}\right) \subset \gamma\left(L i S_{\phi_{n}}\right)=\left\{\gamma(x): x \in L i S_{\phi_{n}}\right\}=\operatorname{Li} \phi_{n}
$$

This completes the proof of (4.7). Since by assumption $\phi_{n}(t) \rightarrow \phi(t) \mu-$ a.e., i.e., $\phi(t)=L i \phi_{n}(t)=L s \phi_{n}(t) \mu-$ a.e., it follows from Lemma 4.5 and the expression (4.7) above that

$$
\int \phi=\int L i \phi_{n} \subset \operatorname{Li} \int \phi_{n} \subset \operatorname{Ls} \int \phi_{n} \subset \operatorname{cl} \int L s \phi_{n}=\operatorname{cl} \int \phi .
$$

Therefore,

$$
\operatorname{cl} \int_{T} \phi(t) \mathrm{d} \mu(t)=L i \int_{T} \phi_{n}(t) \mathrm{d} \mu(t)=L s \int_{T} \phi_{n}(t) \mathrm{d} \mu(t),
$$

i.e.,

$$
\int_{T} \phi_{n}(t) \mathrm{d} \mu(t) \rightarrow \operatorname{cl} \int_{T} \phi(t) \mathrm{d} \mu(t) .
$$


If now $\phi(\cdot)$ is convex valued, (4.8) can be written (recall the second conclusion of Lemma 4.5 ) as

$$
\int \phi=\int \operatorname{Li} \phi_{n} \subset \operatorname{Li} \int \phi_{n} \subset \operatorname{Ls} \int \phi_{n} \subset \int L s \phi_{n}=\int \phi
$$

Thus,

$$
\int_{T} \phi(t) \mathrm{d} \mu(t)=L i \int_{T} \phi_{n}(t) \mathrm{d} \mu(t)=L s \int_{T} \phi_{n}(t) \mathrm{d} \mu(t),
$$

i.e.,

$$
\int_{T} \phi_{n}(t) \mathrm{d} \mu(t) \rightarrow \int_{T} \phi(t) \mathrm{d} \mu(t)
$$

and this completes the proof of Corollary 3.2 .

\section{References}

Artstein, Z., 1979, A note on Fatou's lemma in several dimensions, Journal of Mathematical Economics 6, 277-282.

Aubin, J.-P. and I. Ekeland, 1984, Applied nonlinear analysis (Wiley, New York).

Aumann, R.J., 1965, Integrals of set-valued functions, Journal of Mathematical Analysis and Applications 12, 1-12.

Aumann, R.J., 1966, Existence of a competitive equilibrium in markets with a continuum of agents, Econometrica 34, 1-17.

Aumann, R.J., 1967, Measurable utility and the measurable choice theorem, La Decision (C.N.R.S. Aix-en-Provence) pp. 15-26.

Aumann, R.J., 1976, An elementary proof that integration preserves upper semicontinuity, Journal of Mathematical Economics 3, 15-18.

Balder, E.J., 1984, A unifying note on Fatou's lemma in several dimensions, Mathematics of Operations Research 29, 267-275.

Balder, E.J., 1988, Fatou's lemma in infinite dimensions, Journal of Mathematical Analysis and Applications 136, 450-465.

Balder, E.J. and N.C. Yannelis, 1988, Equilibria in random and Bayesian games with a continuum of players, Mimeo.

Datko, R., 1973, On the integration of set-valued mappings in a Banach space, Fundamenta Mathematicae 78, 205-208.

Debreu, G., 1967, Integration of correspondences, Proceedings of the Fifth Berkeley Symposium on Mathematical Statistics and Probability 2, part 1, 351-372.

Diestel, J., 1977, Remarks on weak compactness in $L_{1}(\mu, X)$, Glasgow Mathematical Journal 18 , 87-91.

Diestel, J. and J.J. Uhl, 1977, Vector measures, Mathematical surveys, no. 15 (American Society, Providence, RI).

Dunford, N. and J.T. Schwartz, 1958, Linear operators, Part I (Interscience, New York).

Hildenbrand, W. and J.-F. Mertens, 1971, On Fatou's lemma in several dimensions, Zeitschrift für Wahrscheinlichkeitstheorie und verwandte Gebiete 17, 151-155.

Himmelberg, C.J., 1975, Measurable relations, Fundamenta Mathematicae 87, 53-72.

Khan, M. Ali, 1986, Equilibrium points of nonatomic games over a Banach space, Transactions of the American Mathematical Society 29, 737-749.

Khan, M. Ali, 1985, On the integration of set-valued mappings in a non-reflexive Banach space, Simon Stevin 59, 257-267.

Khan, M. Ali and M. Majumdar, 1986, Weak sequential convergence in $L_{1}(\mu, X)$ and an approximate version of Fatou's lemma, Journal of Mathematical Analysis and Applications $114,569-573$. 
Kuratowski, K., 1966, Topology, Vol. 1 (Academic Press, New York).

Rustichini, A., 1989, A counterexample and an exact version of Fatou's lemma in infinite dimensions, Archiv der Mathematik 52, 357-362.

Rustichini, A. and N.C. Yannelis, 1986, An elementary proof of Fatou's lemma in finite dimensional spaces, Discussion paper no. 237 (Department of Economics, University of Minnesota, Minneapolis, MN).

Rustichini, A. and N.C. Yannelis, 1988, What is perfect competition?, Mimeo.

Schmeidler, D., 1970, Fatou's lemma in several dimensions, Proceedings of the American Mathematical Society 24, 300-306.

Yannelis, N.C., 1987, Equilibria in non-cooperative models of competition, Journal of Economic Theory $41,96-111$.

Yannelis, N.C., 1988, Fatou's lemma in infinite dimensional spaces, Proceedings of the American Mathematical Society 102, 303-310.

Yannelis, N.C., 1989, Weak sequential convergence in $L_{p}(\mu, X)$, Journal of Mathematical Analysis and Applications 141, 72-83. 